\title{
Function of terahertz spectra in monitoring the decomposing process of biological macromolecules and in investigating the causes of photoinhibition
}

\author{
Yuangang $\mathrm{Qu}^{1,2^{*}}$, Shuai Zhang ${ }^{1}$, Yuji $\operatorname{Lian}^{1} \&$ Tingyun Kuang ${ }^{2}$ \\ ${ }^{1}$ College of Life Sciences, Linyi University, Linyi 276000, China; \\ ${ }^{2}$ Key Laboratory of Photobiology, Institute of Botany, Chinese Academy of Sciences, Beijing 100093, China
}

Received March 11, 2016; accepted August 15, 2016; published online October 31, 2016

\begin{abstract}
Chlorophyll $a$ and $\beta$-carotene play an important role in harvesting light energy, which is used to drive photosynthesis in plants. In this study, terahertz $(\mathrm{THz})$ and visible range spectra of chlorophyll $a$ and $\beta$-carotene and their changes under light treatment were investigated. The results show that the all $\mathrm{THz}$ transmission and absorption spectra of chlorophyll $a$ and $\beta$-carotene changed upon light treatment, with the maximum changes at $15 \mathrm{~min}$ of illumination indicating the greatest changes of the collective vibrational mode of chlorophyll $a$ and $\beta$-carotene. The absorption spectra of chlorophyll $a$ in the visible light region decreased upon light treatment, signifying the degradation of chlorophyll $a$ molecules. It can be inferred from these results that the $\mathrm{THz}$ spectra are very sensitive in monitoring the changes of the collective vibrational mode, despite the absence of changes in molecular configuration. The $\mathrm{THz}$ spectra can therefore be used to monitor the decomposing process of biological macromolecules; however, visible absorption spectra can only be used to monitor the breakdown extent of biological macromolecules.
\end{abstract}

chlorophyll $a, \beta$-carotene, light, terahertz spectra

Citation: Qu, Y., Zhang, S., Lian, Y., and Kuang, T. (2017). Function of terahertz spectra in monitoring the decomposing process of biological macromolecules and in investigating the causes of photoinhibition. Sci China Life Sci 60, 307-312. doi: 10.1007/s11427-016-0057-9

\section{INTRODUCTION}

The thylakoid membranes of plants contain pigments that capture light energy for photosynthesis, including chlorophyll $a$, chlorophyll $b$, and $\beta$-carotene. In vascular plants, chlorophyll $a$ is more abundant than chlorophyll $b$. Chlorophylls are the most abundant pigments in plants and have the most important roles in light harvesting. The tetrapyrrole ring of chlorophylls, called the porphyrin ring, is similar to heme but contains $\mathrm{Mg}^{2+}$. Chlorophylls also possess a hydrophobic phytol side chain. The porphyrin ring, which is hydrophilic, contains a light-absorbing network of conjugated double bonds (Figure 1A). Chlorophyll

*Corresponding author (email: quyuangang@lyu.edu.cn) $a$ and chlorophyll $b$ both absorb light in the violet-to-blue region (maximum absorption at $400-500 \mathrm{~nm}$ ) and the orange-to-red region (maximum absorption 650-700 nm) of the electromagnetic spectrum. Chlorophylls do not absorb much green light; hence they have a green color (Kuczynska et al., 2015). Like chlorophylls, $\beta$-carotenes also contain a series of conjugated double bonds that allow them to capture light (Figure 1B). The absorption maxima of $\beta$-carotene complement those of chlorophylls and thus broaden the range of light energy that can be absorbed by phototrophs (Domonkos and Gombos, 2013). The energy captured by chlorophyll $b$ and $\beta$-carotene is transferred to chlorophyll $a$. All energy absorbed is eventually transferred to a pair of special chlorophyll $a$ molecules in the reaction center of photosystem II (PSII), and then it is used to drive the pho- 

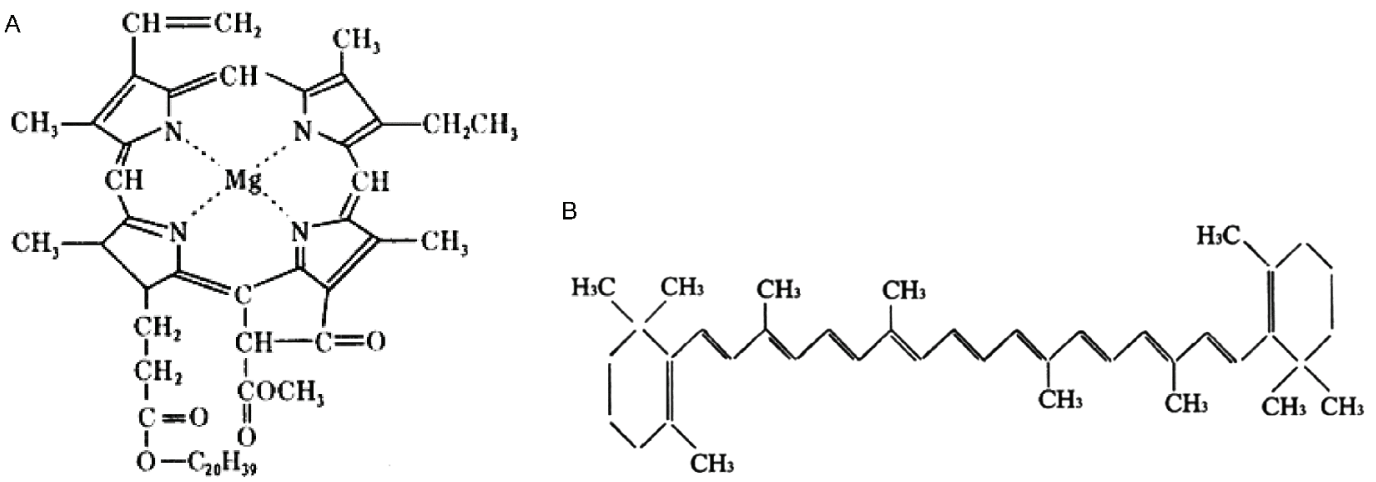

Figure 1 The structure of chlorophyll $a(\mathrm{~A})$ and $\beta$-carotene (B) molecules.

tochemistry reaction (Barber, 1998; Zhang, 2015).

Although sunlight is an essentialenergy source for plant growth, it is an environmental stress factor for plants. Light that is too intense may induce the destruction ("bleaching") of chlorophyll. High light intensities may cause chlorophyll molecules to go to a different excited state. The energy released from these excited chlorophyll molecules is passed to oxygen molecules, which become highly reactive and bleach the chlorophyll (Kingsley et al., 2003). The direct mechanism of this bleaching is not yet well understood.

The use of terahertz time-domain spectroscopy ( $\mathrm{THz}-$ TDS) for studies of conformational flexibility and conformational change in biomolecules has attracted great interest (Markelz et al., 2002; Whitmire et al., 2003; Wilmink and Grundt, 2011). Many conformational mode dynamics of biomolecules occur on the picosecond time scale, and these large-scale structural transitions cause the collective vibrational mode changes that can be detected in the far-infrared or terahertz (THz) frequency range (Markelz et al., 2002; Xie et al., 2014). THz-TDS has been performed to distinguish different molecular species, mutated species, and conformation change of biomolecules (Whitmire et al., 2003; Zhang et al., 2015).

As biomolecules, the collective vibrational mode changes of photosynthesis pigments can also reflect their conformational changes. Energy absorbance and energy transfer between pigments is affected by their conformation. Hence, studying the collective vibrational mode of these pigments is directly relevant to photosynthesis research. In this experiment, THz-TDS was used to study the collective vibrational mode changes of chlorophyll $a$ and $\beta$-carotene under extreme light stress, with the aim of investigating the causes of photoinhibition and THz-TDS use in photosynthesis research. Chlorophyll $b$ was not used because of its similar structure with chlorophyll $a$. It was found that the $\mathrm{THz}$ spectra were very sensitive to changes of the collective vibrational mode of a molecule, despite no changes occurring in the molecular configuration. The $\mathrm{THz}$ spectra can be used to monitor the decomposing process of biological macromolecules; however, visible absorption spectra can only be used to monitor the extent $\mathrm{b}$ of reakdown of biological macromolecules. Photoinhibition often occurs when plants are exposed to high light intensity stress, but the mechanism of photoinhibition is not clear. In this study, it was found that the collective vibrational mode of chlorophyll $a$ and $\beta$-carotene both changed under high light intensity. As these changes affect heat dissipation and energy transfer, it is suggested that changes of the collective vibrational mode may be one of the causes of photoinhibition.

\section{RESULTS}

\section{Changes of the time-domain THz waveforms of chloro- phyll $a$ and $\beta$-carotene induced by illumination}

The time-domain $\mathrm{THz}$ waveforms of the light $\left(1,000 \mu \mathrm{E} \mathrm{m}{ }^{-2}\right.$ $\mathrm{s}^{-1}$ )-illuminated and intact chlorophyll $a$ and $\beta$-carotene are shown in Figure 2. The waveforms show that the response times of both chlorophyll $a$ and $\beta$-carotene are slower than that of polyethylene. The extent of the changes in response time for $\beta$-carotene is greater than that for chlorophyll $a$ upon light treatment.

\section{Changes of the transmission spectra of chlorophyll $a$ and $\beta$-carotene induced by illumination}

The temporal waveforms shown in Figure 2 were further converted into the frequency-domain amplitude by fast Fourier transform shown in Figure 3. Figure 3A and B shows that the transmission spectrum of chlorophyll $a$ shifts to a lower frequency under illumination $\left(1,000 \mu \mathrm{E} \mathrm{m}^{-2} \mathrm{~s}^{-1}\right)$ for $5 \mathrm{~min}$. Then the spectrum returns to the intact state (0 min) at 10-min illumination. After $10 \mathrm{~min}$, the spectrum shifts to a lower frequency again, and the greatest shift occurs in the curve of $15 \mathrm{~min}$. Figure $3 \mathrm{C}$ and $\mathrm{D}$ shows that the transmission spectra of $\beta$-carotene also shifts to a lower frequency when under illumination. The greatest shift occurs in the curve of $15 \mathrm{~min}$. After this, the spectra shift back towards a higher frequency. Changes of the $\mathrm{THz}$ transmission spectra signify changes of the collective vibrational mode of chlorophyll $a$ and $\beta$-carotene. 

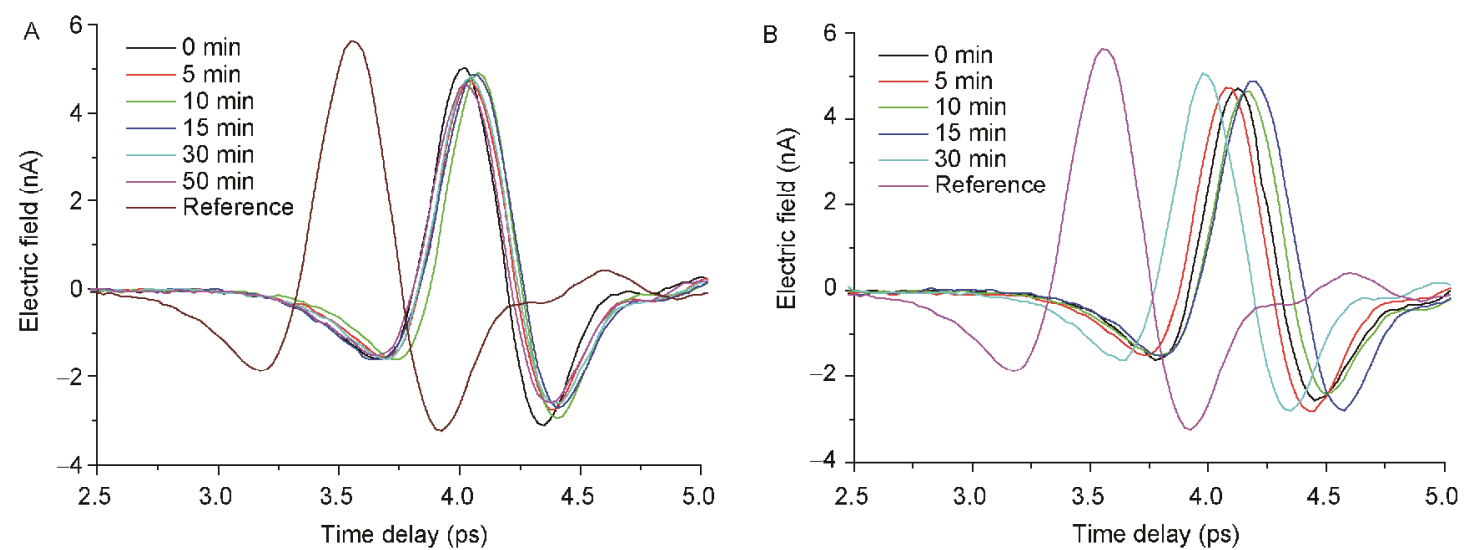

Figure 2 The time-domain THz waveforms of chlorophyll $a$ (A) and $\beta$-carotene (B). The light intensity used was $1,000 \mu \mathrm{E} \mathrm{m}^{-2} \mathrm{~s}^{-1}$. The illumination times were $0,5,10,15,30$, and $50 \mathrm{~min}$. The reference curve was obtained from polyethylene.
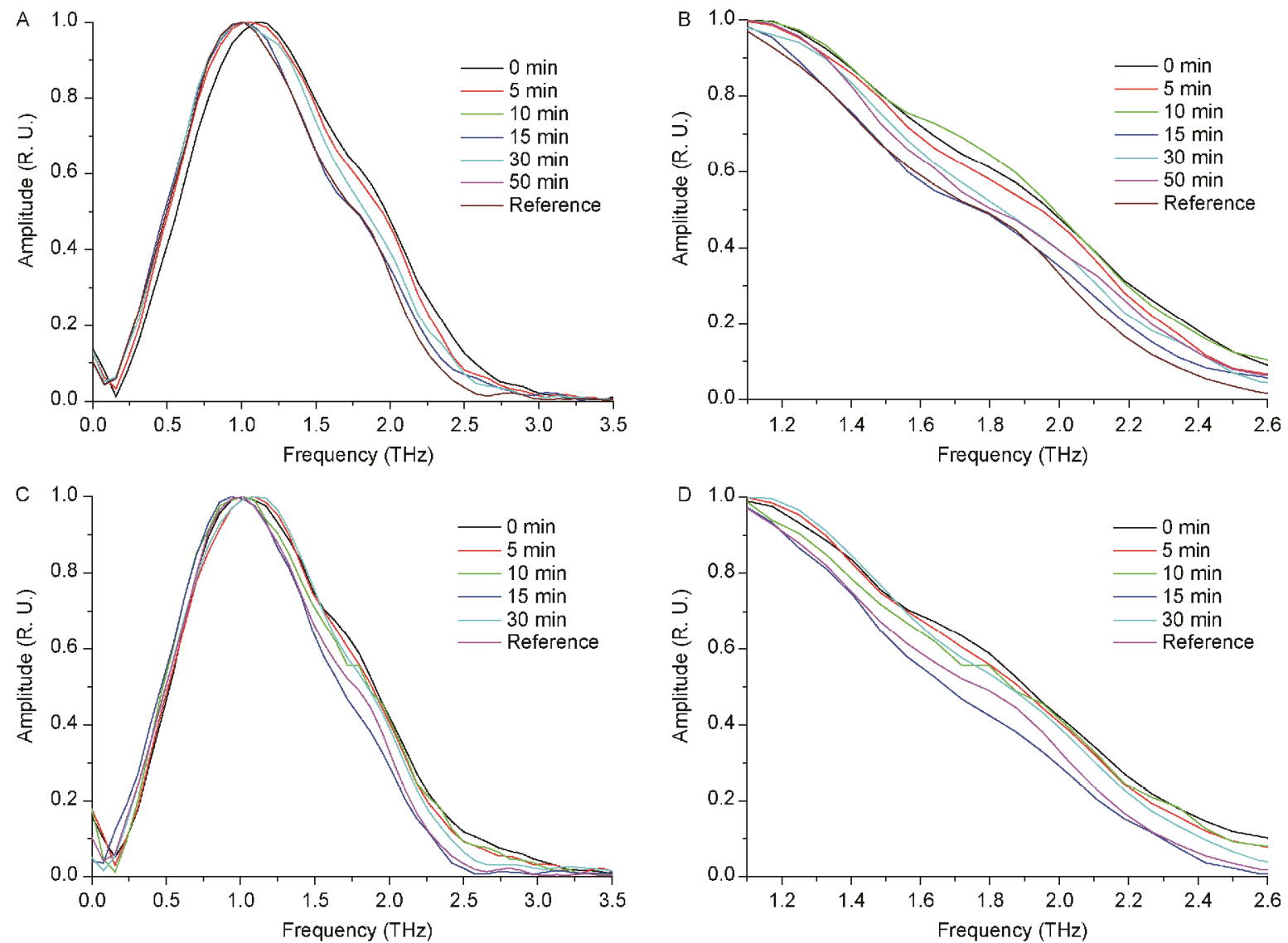

Figure 3 Fourier transform spectra of chlorophyll $a$ (A and B) and $\beta$-carotene (C and D). B and D is an enlargement of A and C. The light intensity used was $1,000 \mu \mathrm{E} \mathrm{m}^{-2} \mathrm{~s}^{-1}$. For chlorophyll $a$, the illumination times were $0,5,10,15,30$, and $50 \mathrm{~min}$. For $\beta$-carotene, the illumination times were $0,5,10,15$, and $30 \mathrm{~min}$. The reference curve was obtained from polyethylene. The data were normalized to the peak value of the reference spectrum.

\section{Changes of the absorption spectra of chlorophyll $a$ and $\beta$-carotene induced by illumination}

The absorbance of the samples can be expressed as

$$
E_{\mathrm{s}}(\omega) / E_{\mathrm{r}}(\omega)=T(\omega) \exp (-\alpha d+i(n \omega d / c)),
$$

where $T(\omega)$ is a factor related to the reaction at the sam-
ple/PE window interfaces, $d$ is the sample thickness, $\alpha$ is the power absorption coefficient, $n$ is an index of the sample, and $c$ is the light velocity in a vacuum. Figure 4A shows the $\mathrm{THz}$ absorption spectra of chlorophyll $a$ from $0.25-2 \mathrm{THz}$. The gradient of the curve for $5 \mathrm{~min}$ of illumination $\left(1,000 \mu \mathrm{E} \mathrm{m}^{-2} \mathrm{~s}^{-1}\right)$ decreases relative to the intact curve, and returns to the initial state for the curve for 10-min 

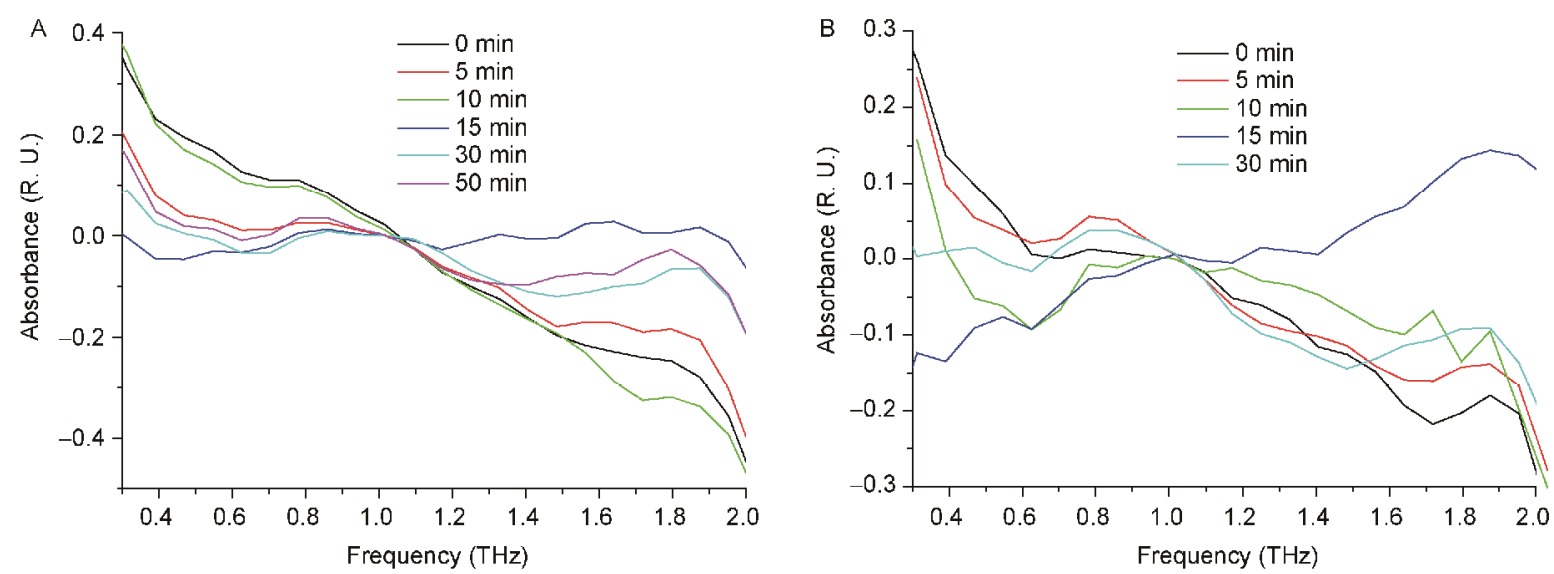

Figure $4 \mathrm{THz}$ absorption spectra of chlorophyll $a$ (A) and $\beta$-carotene (B) during $0.25-2 \mathrm{THz}$. The light intensity used was $1,000 \mu \mathrm{E} \mathrm{m} \mathrm{m}^{-2} \mathrm{~s}^{-1}$. The illumination times were $0,5,10,15,30$, and $50 \mathrm{~min}$.

illumination. After $10 \mathrm{~min}$ of illumination, the gradient decreases again, and become roughly flat at the 15 -min point. In addition, the curve for 30- and 50-min illumination are very similar. Figure $4 \mathrm{~B}$ shows the $\mathrm{THz}$ absorption spectra of $\beta$-carotene from $0.25-2 \mathrm{THz}$. Upon illumination, the curve does not show much change up to $15 \mathrm{~min}$, but the gradient increases when $\beta$-carotene is illuminated for about $15 \mathrm{~min}$, and then it is reverts to the initial state when $\beta$-carotene is illuminated for more than $15 \mathrm{~min}$. Changes of the $\mathrm{THz}$ absorption spectra also translate to changes of the collective vibrational mode of chlorophyll $a$ and $\beta$-carotene.

\section{Light-induced changes of the absorption spectra of chlorophyll $a$ in visible light region}

Figure 5 shows that the absorption spectra of chlorophyll $a$ in the visible light region has two peaks at 432 and $660 \mathrm{~nm}$. Upon light treatment $\left(1,000 \mu \mathrm{E} \mathrm{m}^{-2} \mathrm{~s}^{-1}\right)$ treatment, both peaks decrease. The peak value at $660 \mathrm{~nm}$ decreases by $11 \%, 26 \%, 40 \%, 48 \%, 55 \%, 65 \%$, and $85 \%$ when chlorophyll $a$ is illuminated for $0,2,5,10,15,20,30$, and $60 \mathrm{~min}$, respectively. In addition, the green color of chlorophyll $a$ disappeared gradually when illuminated (Figure 6). For chlorophyll $a$, it is the tetrapyrrole ring, known as the head section, that can absorb visible light. Therefore, these results indicate that the head section was degraded continuously with the illumination.

\section{DISCUSSION}

The degradation of chlorophyll $a$ upon illumination (Figures 5 and 6) suggest that visible absorption spectra can monitor the extent of breakdown of biological macromolecules.

It is reported (Walther et al., 2002; Korter et al., 2005), and can be understood from fundamental physics principles, that the smaller the moving mass, the higher the vibrational frequencies for the same kind of molecule. Figure $3 \mathrm{~A}$ and $\mathrm{B}$

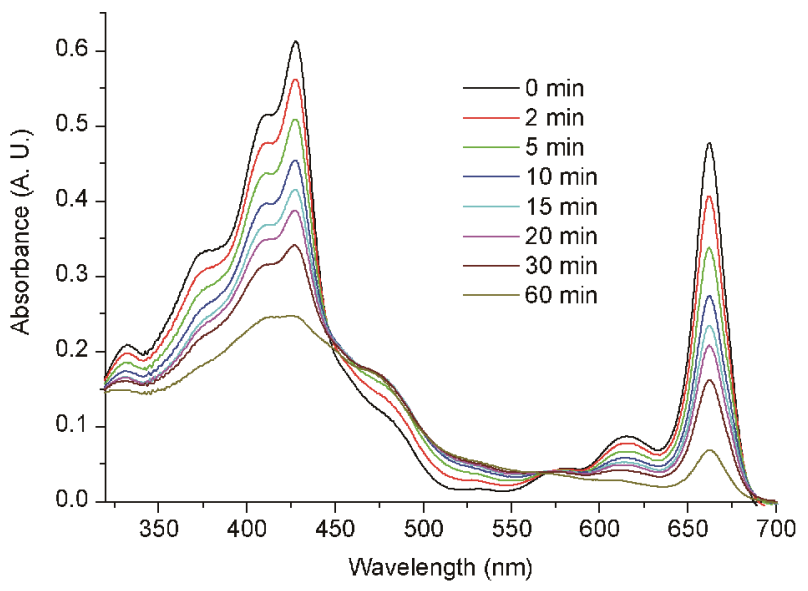

Figure 5 Absorption spectra changes of chlorophyll $a$ in the visible light region when illuminated by strong white light of $1,000 \mu \mathrm{E} \mathrm{m}^{-2} \mathrm{~s}^{-1}$. Spectra from top down correspond to samples irradiated for $0,2,5,10,15,20,30$, and $60 \mathrm{~min}$.

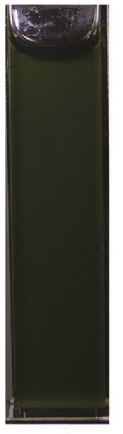

A

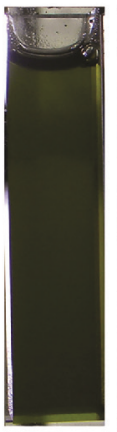

B

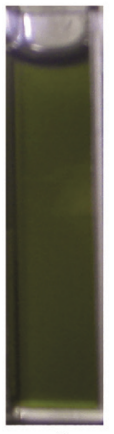

C

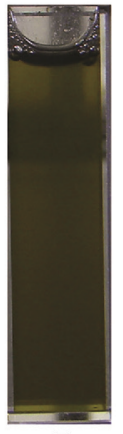

D

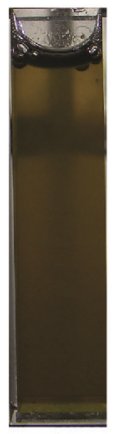

$\mathrm{E}$
Figure 6 The color changes of chlorophyll $a$ when illuminated by strong white light of $1,000 \mu \mathrm{E} \mathrm{m} \mathrm{m}^{-2} \mathrm{~s}^{-1}$. A, B, C, D and E show the sample color after $0,5,15,30$, and $60 \mathrm{~min}$ of illumination, respectively.

shows that the transmission spectrum of chlorophyll $a$ shifts to the low frequency during illumination. This implies that chlorophyll $a$ is degraded to different molecules from chlo- 
rophyll $a$ itself, but not only the side chain of chlorophyll $a$ is shortened. HÖrtensteiner reported that, under strong light treatment, chlorophyll a is broken down into chlorophyllide $a$, pheophorbide $a$, red chlorophyll catabolite (RCC), primary fluorescent chlorophyll catabolite (pFCC), nonfluorescent chlorophyll catabolites (NCCs), and monopyrroles (HÖrtensteiner, 2006). The different transmission spectra in Figure $3 \mathrm{~A}$ and $\mathrm{B}$ are thought to be caused by the different breakdown products. The product indicated by the $10-\mathrm{min}$ curve may have a similar transmission spectrum with the intact chlorophyll $a$ molecule, so the curve at 10 min returns to the state of $0 \mathrm{~min}$. These results suggest that the $\mathrm{THz}$ transmission spectra can be used to monitor the decomposing process of biological macromolecules, and especially to monitor the formation of new molecules even if they have not been identified. In fact, it can be seen from Figure 4A that $\mathrm{THz}$ absorption spectra can also have this kind of monitoring function, unlike the visible absorption spectra as discussed above.

$\beta$-carotene is one of the most stable natural pigments (Zhang et al., 2014). Under the experimental conditions of this study, the visible absorption spectra of $\beta$-carotene did not change (data not shown). This indicates that the conjugated double bonds of $\beta$-carotene are not broken. Figure $3 \mathrm{C}$ and $\mathrm{D}$ and Figure 4B show that the transmission and absorption spectra of $\beta$-carotene in the $\mathrm{THz}$ region all changed. This means that the collective vibrational mode of $\beta$-carotene changed even though the configuration did not change under illumination conditions. It implies that the $\mathrm{THz}$ spectra are very sensitive to changes of the collective vibrational mode, despite no changes occurring in the molecular configuration.

Exposure of plant leaves to light levels in excess of what can be utilized in photosynthesis often results in a decline in photosynthetic activity or destruction in the photosynthetic apparatus. This high-intensity light effect is known as photoinhibition (Allahverdieva and Aro, 2012; Ohad et al., 2011). Numerous experiments prove that photoinhibition can also be induced by low light (Tyystjärvi, 2013). Why photoinhibition happens is not very clear so far, but it occurs mainly in PSII. The light energy absorbed by the PSII antenna chlorophyll molecules can be dissipated as fluorescence, as heat by non-radiative dissipation, by transfer to photosystem I (PSI), and in photochemical activity by PSII (Björkman, 1987). There is evidence that photoinhibition of photosynthesis in vivo may be caused by increased thermal dissipation of excited chlorophylls at the antenna or the reaction center levels (Öquist, 1988). The results of this study show that the molecular vibration mode change upon illumination. In terms of thermodynamics, changes of the molecular vibration mode correspond to that of the thermal energy, but not to fluorescence or other factors. Thus, illumination may affect the thermal dissipation process of pigments, and thereby the photoinhibition. Whether this effect is mediated by up- or down-regulation remains to be studied further.

Förster resonance energy transfer (FRET) is a mechanism describing energy transfer between two light-sensitive molecules (chromophores). A donor chromophore, initially in its electronic excited state, may transfer energy to an acceptor chromophore through nonradiative dipole-dipole coupling (Cheng, 2006). This mechanism of energy transfer involves intramolecular and intermolecular (aggregate normal-mode) vibrations (May and Kühn, 2011). When illuminated, the collective vibrational mode of chlorophyll $a$ and $\beta$-carotene both change. This implies that high light intensity may affect the energy transfer among pigments, thus being one of the causes of photoinhibition.

\section{MATERIALS AND METHODS}

\section{Materials}

Chlorophyll $a$ and $\beta$-carotene were purchased from Sigma. The chlorophyll $a$ and $\beta$-carotene were dissolved in $80 \%$ acetone and hexane, respectively.

\section{White-light illumination}

A halogen lamp was used for illumination of the temperature-controlled vessel containing the suspension of chlorophyll $a$ or $\beta$-carotene through a filter with circulating cold water. The white-light intensity at the surface of the vessel was $1,000 \mu \mathrm{E} \mathrm{m}^{-2} \mathrm{~s}^{-1}$. The light treatment was carried out at room temperature at a concentration of $0.2 \mathrm{mg} \mathrm{mL}^{-1}$ of pigments, and with continuous stirring.

\section{Absorption measurement}

The absorption spectra in the visible range were recorded with a UV-3000 spectrophotometer (Hitachi, Japan) at room temperature.

\section{THz-TDS}

Before the THz-TDS measurement, the chlorophyll $a$ and $\beta$-carotene solutions were first mixed with polyethylene (PET) powder to create two mixtures. Since PET is nearly transparent in the $\mathrm{THz}$ waveband it is a suitable filling material for spectroscopic applications in this spectral region. For each sample, $50 \mu \mathrm{L}$ of sample solution was put into $88 \mathrm{mg}$ PET powder that was held in a disk-like cell with high-density polyethylene windows. A thin metallic rod was used to stir the mixture until it was uniformly mixed. Then an electric blower was used to vaporize the moisture in the mixture. Finally, the mixture was compressed into a disk approximately $10 \mathrm{~mm}$ in diameter and $1.5 \mathrm{~mm}$ in thickness. All the THz-TDS measurements were conducted in a vacuum chamber to avoid the interference of water vapor and to ensure the sample remained in dry conditions. For each measurement, a reference waveform was taken by replacing the sample with PET powder. 
A standard THz-TDS configuration was used in our study. The $\mathrm{THz}$ radiation was generated by a piece of (100)-oriented InAs wafer, which was excited by optical pulses from a mode-locked Ti-sapphire laser $(\lambda=800 \mathrm{~nm}$, $\tau_{\text {FWHM }}=100 \mathrm{fs}$ ). The $\mathrm{THz}$ wave was collected and aligned using a pair of paraboloid mirrors before being focused onto the sample. After passing through the sample, the $\mathrm{THz}$ wave was re-aligned and then detected via the electro-optic effect in a (110)-oriented ZnTe crystal. All the acquired samples and reference waveforms were converted to their Fourier transforms $E_{\mathrm{s}}(\omega)$ and $E_{\mathrm{r}}(\omega)$, and the transmission spectra were obtained as a ratio of their Fourier spectra, which is $E_{\mathrm{s}}(\omega) / E_{\mathrm{r}}(\omega)$. In this way, the complex system response was simply eliminated in this way. The multi-reflection pulses could be well resolved temporally, and therefore the spectral interference resulting from the etalon effect was ruled out by truncation of the reflected pulses.

Compliance and ethics The author(s) declare that they have no conflict of interest.

Acknowledgements This work was supported by the Shandong Provincial Natural Science Foundation, China (ZR2012CM020), and the National Training Programs of Innovation and Entrepreneurship for Undergraduates (201410452012).

Allahverdieva, Y., and Aro, E.M. ( 2012). Photosynthetic responses of plants to excess light: mechanisms and conditions for photoinhibition, excess energy dissipation and repair. In Photosynthesis: Plastid Biology, Energy Conversion and Carbon Assimilation. Advances in Photosynthesis and Respiration, J.J. Eaton-Rye, B.C. Tripathy, and T.D. Sharkey, eds. (Dordrecht: Springer), pp. 275-297.

Barber, J. (1998). Photosystem two. Biochim Biophys Acta 1365, 269-277.

Björkman, O. (1987). High-irradiance stress in higher plants and interaction with other stress factors. In Progress in Photosynthesis Research, J. Biggins, ed. (Dordrecht: Springer), pp. 11-18.

Cheng, P. (2006). The Contrast formation in optical microscopy. In
Handbook of Biological Confocal Microscopy, B.P., James, ed. (New York: Springer), pp. 162-206.

Domonkos, I., Kis, M., and Gombos, Z. (2013). Carotenoids, versatile components of oxygenic photosynthesis. Prog Lipid Res 52, 539-561.

HÖrtensteiner, S. (2006). Chlorophyll degradation during senescence. Annu Rev Plant Biol 57, 55-77.

Kingsley, R.S., Shelley, J., and James, E.B. (2003). photosynthesis. In Introductory Plant Biology, R.S. Kingsley, J. Shelley, and E.B. James, ed. (New York: McGraw-Hill Companies), pp. 172-186.

Korter, T.M., Balu, R., Campbell, M.B., Beard, M.C., Gregurick, S.K., and Heilweil, E.J. (2005). Terahertz spectroscopy of solid serine and cysteine. Chem Phys Lett 418, 65-70.

Kuczynska, P., Jemiola-Rzeminska, M., and Strzalka, K. (2015). Photosynthetic Pigments in Diatoms. Mar Drugs 13, 5847-5881.

Zhang, L., Zuo, J., and Zhang, C. (2014). Observation of terahertz spectra of all-trans $\beta$-Carotene. Spectrosc Spect Anal 34, 405-406.

Markelz, A., Whitmire, S., Hillebrecht, J., and Birge, R. (2002). THz time domain spectroscopy of biomolecular conformational modes. Phys Med Bio 21, 3797-3805.

May, V., and Kühn, O. (2011). Charge and Energy Transfer Dynamics in Molecular Systems (Weinheim: Wiley-VCH Verlag GmbH \& Co.).

Ohad, I., Berg, A., Berkowicz, S.M., Kaplan, A., and Keren, N. (2011). Photoinactivation of photosystem II: is there more than one way to skin a cat? Physiol Plantarum 142, 79-86.

Öquist, G. (1988). Stress and adaptation in photosynthesis. In Light in Biology and Medicine, R.H. Douglas, J. Moan, and F.D. Acqua, ed. (New York: Plenum Press), pp. 433-440.

Tyystjärvi, E. (2013). Photoinhibition of photosystem II. Int Rev Cel Mol Bio 300, 243-302.

Walther, M., Plochocka, P., Fischer, B., Helm, H., and Jepsen, P. Uhd. (2002). Collective vibrational modes in biological molecules investigated by terahertz timedomain spectroscopy. Biopolymers 67, 310-313.

Whitmire, S.E., Wolpert, D., Markelt, A.G., Hillebrecht, J.R., Galan, J., and Birge, R.R. (2003). Protein flexibility and conformational state: a comparison of collective vibrational modes of wild-type and D96N bacteriorhodopsin. Biophys J 85, 1269-1277.

Wilmink, G.J., and Grundt, J.E. (2011). Invited review article: current state of research on biological effects of terahertz radiation. J Infrared Millm W 32, 1074-1122.

Xie, L., Yao, Y. and Ying, Y. (2014). The application of terahertz spectroscopy to protein detection. Appl Spectrosc Rev 49, 448-461.

Zhang, C. (2015). The first artificial $\mathrm{Mn}_{4} \mathrm{Ca}$-cluster mimicking the oxygen-evolving center in photosystem II. Sci China Life Sci 58, 816-817.

Zhang, W., Nie, J., and Tu, S. (2015). Study on identification methods in the detection of transgenic material based on terahertz time domain spectroscopy. Opt Quant Electron 47, 3533-3543.

Open Access This article is distributed under the terms of the Creative Commons Attribution License which permits any use, distribution, and reproduction in any medium, provided the original author(s) and source are credited. 